\title{
A spectrally selective gap surface-plasmon-based nanoantenna emitter compatible with multiple thermal infrared applications
}

\author{
Ataollah Kalantari Osgouei ${ }^{1,2, *} \mathbb{D}$, Amir Ghobadi ${ }^{1,3}$, Bahram Khalichi ${ }^{1,3}$ (D) \\ and Ekmel Ozbay ${ }^{1,2,3,4, *}$ \\ ${ }^{1}$ NANOTAM-Nanotechnology Research Center, Bilkent University, 06800 Ankara, Turkey \\ ${ }^{2}$ Department of Physics, Bilkent University, 06800 Ankara, Turkey \\ ${ }^{3}$ Department of Electrical and Electronics Engineering, Bilkent University, 06800 Ankara, Turkey \\ ${ }^{4}$ UNAM-Institute of Materials Science and Nanotechnology, Bilkent University, Ankara, Turkey \\ E-mail: akalantari@bilkent.edu.tr and ozbay@bilkent.edu.tr
}

Received 8 April 2021, revised 21 June 2021

Accepted for publication 20 July 2021

Published 20 August 2021

\begin{abstract}
Wavelength-selective nanoantenna emitters have attracted considerable attention due to their widespread applications ranging from thermal radiation management to thermophotovoltaics. In this paper, we design a wavelength-selective nanoantenna emitter based on the excitation of gap-surface plasmon modes using a metal-insulator-metal configuration (silicon dioxide $\left(\mathrm{SiO}_{2}\right)$ sandwiched between silver ( $\mathrm{Ag}$ ) layers) for satisfying multiple infrared applications. The proposed design, which is called design I, realizes triple narrowband perfect absorptions at the resonance wavelengths of $1524 \mathrm{~nm}, 2279 \mathrm{~nm}$, and $6000 \mathrm{~nm}$, which perfectly match the atmospheric absorption bands while maintaining relatively low emissivity in the atmospheric transparency windows of 3-5 $\mu \mathrm{m}$ and $8-12 \mu \mathrm{m}$. Later, the functionality of design I is extended, which is called design II, to include a broadband absorption at the near-infrared region to minimize the solar irradiation reflection from the nanoantenna emitter. Finally, singleand three-layer graphene are introduced to provide a real-time tuning of the infrared signature of the proposed nanoantenna emitter (design II). It is also demonstrated that the three-layer graphene structure can suppress an undesired absorption resonance wavelength related to the intrinsic vibrational modes (optical phonons) of the $\mathrm{SiO}_{2}$ layer by $53.19 \%$ compared to $25.53 \%$ for the single-layer one. The spectral analysis of design I is validated using both analytical and numerical approaches where the numerical simulation domain is extended for the analysis of design II. The thermal characteristic analyses of design I and design II (without/with graphene layers) reveal that infrared signatures of the blackbody radiation are significantly reduced for the whole wavelength spectrum at least by $96 \%$ and $91 \%$ within a wide temperature ranging from room temperature to $500 \mathrm{~K}$, respectively.
\end{abstract}

Keywords: nanoantenna emitter, wavelength selectivity, plasmonic structure, gap surface plasmon, thermal radiation management, solar absorber

(Some figures may appear in colour only in the online journal)

* Authors to whom any correspondence should be addressed. 


\section{Introduction}

Total emissive power per unit area from a hot surface is defined by the Stefan-Boltzmann law $P=\varepsilon \sigma T^{4}[1,2]$, where $\sigma$ is the Stefan-Boltzmann constant, $\varepsilon$ and $T$ are the emissivity and absolute temperature of the object, respectively. At the thermal equilibrium, Kirchhoff's radiation law $[1,2]$ states that the wavelength-dependent thermal radiation of a surface is equal to the optical absorption of that surface defined as $\varepsilon(T, \lambda)=$ $\alpha(T, \lambda)$. Therefore, suppressing the thermal radiation can be achieved by reducing the emissivity/absorptivity $(\varepsilon)$ or the real temperature of the object $(T)$. However, the poor efficiency of the thermal radiation contributes to a sharp increase in the real temperature of the object. Accordingly, wavelengthselective thermal emitters are widely used to overcome this problem. In particular, a wavelength-selective material-based shield should have a low emissivity to reduce the object's thermal radiation within specific atmospheric windows as well as have a high emission outside of those atmospheric windows for the radiative cooling applications. In other words, the thermal radiation should dominantly be suppressed within the mid-wave infrared (MWIR: 3-5 $\mu \mathrm{m}$ ) and the long-wave infrared (LWIR: 8-12 $\mu \mathrm{m}$ ) ranges representing the transmission channels for electromagnetic waves in the atmosphere, while the thermal radiation is allowed to pass in the nontransmissive (absorption) windows, including 1.5-1.61 $\mu \mathrm{m}$, 2.15-3 $\mu \mathrm{m}, 4-4.2 \mu \mathrm{m}$, and 5-8 $\mu \mathrm{m}$, due to the fact that the absorption phenomenon quench the propagation of emitted photons in these ranges [3]. This wavelength-selective characteristic makes the object blend into the background and offers thermal radiation management for multiple thermal camouflage scenarios. Moreover, to have a comprehensive infrared characteristic, the design should absorb (solar) photons in the range of $0.9-1.7 \mu \mathrm{m}$ to hide from short-wave infrared (SWIR) and laser tracking cameras (typically operating at $1064 \mathrm{~nm}$ ).

Since the design of wavelength-selective thermal nanoantenna emitter is equivalent to the design of wavelengthselective thermal nanoantenna absorber (Kirchhoff's law), metamaterial-based perfect absorbers with wavelength selectivity characteristic have attracted considerable attention due to their potential applications in radiative cooling $[4,5]$ and thermophotovoltaics [6, 7]. In addition, plasmonic metamaterials [8-10] and photonic crystals $[11,12]$ are widely exploited in thermal radiation management [13-18] as an ability to hide or disguise an object from infrared (IR) detection equipment and thermal imaging systems. Although photonic crystals can be designed to realize high emissivity with a special structure $[11,12]$, the configuration complexity makes photonic crystals less suitable in high-temperature environments due to the thermal stress [17]. On the other hand, plasmonic metamaterials as a periodic array of subwavelength structures can be artificially engineered to achieve exotic properties in terms of perfect absorptions and wavelength selectivity. The absorption can reach near unity at specific wavelengths, enabling the realization of perfect absorptions. Generally, metal-insulator-metal (MIM) plasmonicbased cavity designs are considered as one of the typical configurations for designing near unity wavelength-selective absorbers [19-23]. Properly choosing selective emissive materials for an MIM cavity design and its extensions to composite unit cell $[24,25]$ or multi-layer [26, 27] structures can lead to wavelength-selective metamaterial-based nanoantenna emitters/absorbers satisfying the requirements of multiple thermal infrared applications. Overall, in an ideal architecture, a wavelength-selective thermal nanoantenna emitter should radiatively cool the surface within specific atmospheric absorption windows without being detected by infrared cameras.

In the first part of this paper, a wavelength-selective nanoantenna emitter based on the excitation of gap surface plasmon modes using an MIM configuration (silicon dioxide $\left(\mathrm{SiO}_{2}\right)$ sandwiched between silver ( $\left.\mathrm{Ag}\right)$ layers) is proposed. The proposed structure, called design I, shows triple narrowband perfect absorptions at the resonance wavelengths of $1524 \mathrm{~nm}, 2279 \mathrm{~nm}$, and $6000 \mathrm{~nm}$ that perfectly match the atmospheric absorption bands. Meanwhile, the emissivity of the structure in the wavelength ranges of 3-5 $\mu \mathrm{m}$ and 8-12 $\mu \mathrm{m}$ (atmospheric windows) is suppressed considering the average amount of absorption occurred below 0.10 . Later, we extend the functionality of design I to cover the possible threats in the SWIR range (design II). The results indicate that a near perfect absorption is acquired in a broadband spectrum spanning from $929 \mathrm{~nm}$ to $1472 \mathrm{~nm}$ (SWIR range), together with a narrowband absorption peak at $5750 \mathrm{~nm}$ (atmospheric absorption window). Finally, a real-time tuning of the infrared response using external electrostatic bias is added to the system by embedding graphene layers. It is also shown that an undesired resonance wavelength at the absorption spectrum related to the intrinsic vibrational modes (optical phonons) of the $\mathrm{SiO}_{2}$ layer within the LWIR range is reduced by adding a single-layer graphene. Moreover, the amount of reduction is significantly enhanced in the case of embedding a three-layer graphene. In other words, the amount of absorption of the LWIR response for the three-layer graphene device is decreased by $53.19 \%$, compared to $25.53 \%$ for the single-layer graphene one. The performances of the proposed structures are validated using rigorous coupled-wave analysis (RCWA) as an analytical method [28], besides a finitedifferences time domain solver (FDTD) as a numerical method [29]. In addition, the thermal characteristic performances of design I and design II (without/with graphene layers) are validated by calculating spectral radiant emittance at different temperatures.

\section{Design I}

\subsection{Results and discussions}

The schematic diagram of the proposed nanoantenna emitter (design I) is shown in figure 1(a). The unit cell consists of two metallic silver (Ag) layers: a bottom reflector (optically thick with no transmission in our analysis range) and a top grating array, with an insulator layer of $\mathrm{SiO}_{2}$ (silicon dioxide) sandwiched in between. The Ag film is selected as the primary thermal radiation layer because of its unique radiation 
(a)

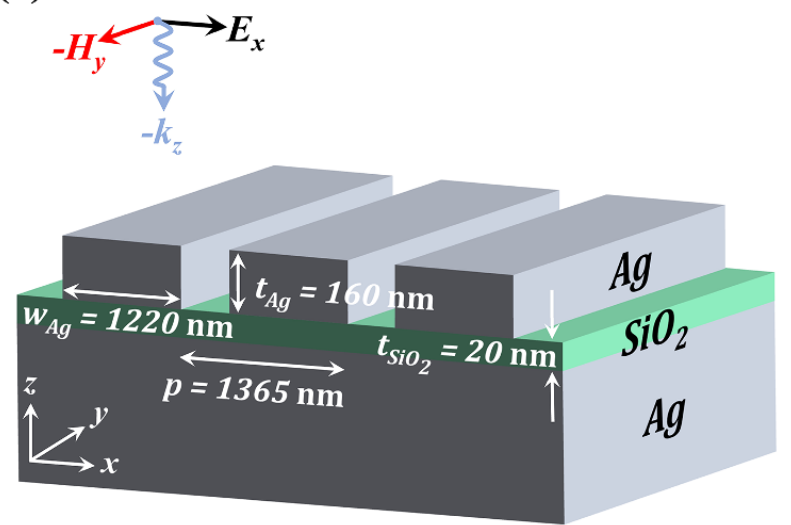

(b)

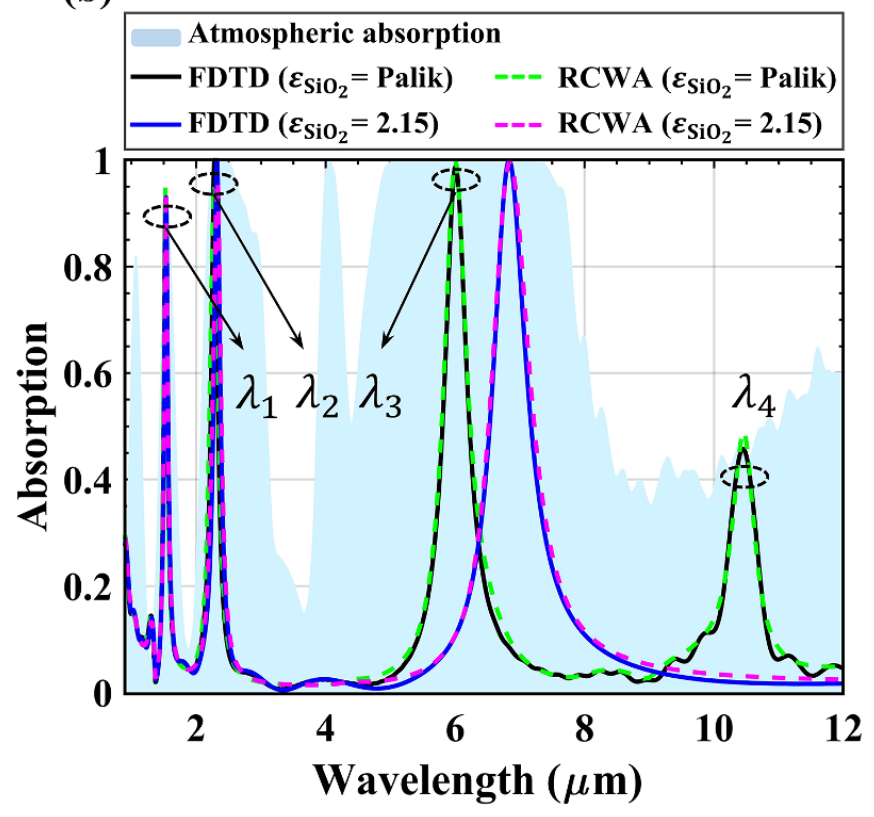

Figure 1. (a) Schematic unit cell of the proposed

wavelength-selective nanoantenna emitter (design I). (b) Spectral absorption responses of design I obtained by considering constant and frequency-dependent values for the permittivity of the $\mathrm{SiO}_{2}$ layer. The atmospheric absorption band [shown as the light blue area] is modeled by the US standard atmosphere compositions at the vertical distance of $5 \mathrm{~km}$.

properties [17], while the $\mathrm{SiO}_{2}$ spacer layer is added to match the selective emissions to the impedance of the air. The analytical (RCWA) and numerical (FDTD) approaches are utilized to investigate the optical properties of the proposed nanoantenna emitter, when the structure is normally illuminated by an $x$-polarized ( $p$-polarization) uniform plane-wave propagating along the $-z$ direction. In the simulations, the frequencydependent refractive indices of $\mathrm{Ag}$ and $\mathrm{SiO}_{2}$ are taken into account from the CRC Handbook of Chemistry and Physics [30] and the Palik model database [31], respectively. Then, based on the utilized simulation approaches, the geometrical parameters as explicitly presented in figure 1(a) are optimized in such a way that the excited resonances in the absorption spectrum match the atmospheric absorption windows. As a result, the thickness of the top nanograting Ag layer is
$t_{\mathrm{Ag}}=160 \mathrm{~nm}$ while its width is considered $w_{\mathrm{Ag}}=1220 \mathrm{~nm}$, and the thickness of the $\mathrm{SiO}_{2}$ layer is set to be $t_{\mathrm{SiO}_{2}}=20 \mathrm{~nm}$. The period of the unit cell is considered to be $p=1365 \mathrm{~nm}$ along the $x$ direction. The absorptivity of design I with respect to the wavelength is presented in figure 1(b), where the analytical and numerical approaches are in good agreement with each other. The results unveil that the fourth resonance $\left(\lambda_{4}\right.$ in figure 1(b)) is due to intrinsic vibrational modes (optical phonons) of the $\mathrm{SiO}_{2}$ layer in the LWIR range [32] since the resonance is suppressed by considering a constant relative permittivity $\left(\varepsilon_{\mathrm{SiO}_{2}} \simeq 2.15\right)$ valid below the wavelength of $8 \mu \mathrm{m}$, as shown in figure 1(b). The other triple narrowband perfect absorptions are successfully achieved at the resonant peaks of $\lambda_{1}=1524 \mathrm{~nm}, \lambda_{2}=2279 \mathrm{~nm}$, and $\lambda_{3}=6000 \mathrm{~nm}$ (see figure 1(b)). These resonances are in very good agreement with the atmospheric spectrum (shown as the light blue area in figure 1(b)), while showing remarkable low average emissivity in the atmospheric windows (i.e. $\varepsilon_{3-5 \mu \mathrm{m}}$ and $\varepsilon_{8-12 \mu \mathrm{m}} \leqslant$ $0.10)$. Therefore, the thermal radiation of the wavelengthselective design can be greatly absorbed by the atmosphere, which makes the coated object hidden to infrared detectors. The atmospheric spectrum is obtained from [33] where the atmospheric absorption is modeled by considering the US standard atmosphere compositions at the vertical distance of $5 \mathrm{~km}$.

\subsection{Physical mechanism}

The physical behavior of design I is analyzed at the resonance wavelengths to determine the types of supported modes by the structure. Therefore, the calculated total electric- and magnetic-field distributions are presented in figures 2(a)-(f). At the resonance wavelengths of $\lambda_{1}, \lambda_{2}$, and $\lambda_{3}$ shown in figures 2(a)-(f), the presented electric and magnetic mode profiles are mostly concentrated within the thin spacer layer, due to the formation of gap surface plasmon (GSP) resonances [34-39]. The GSP resonator, being composed of a thin $\mathrm{SiO}_{2}$ layer sandwiched between the top Ag grating array and bottom Ag substrate, experiences multiple reflections from the termination of the MIM structure. This results in a lateral standing-wave type resonance between the metallic terminations and oscillating antiparallel electric currents in the two metallic layers [34]. Therefore, GSP resonances can be described by a simple Fabry-Perot (FP) resonator formula $[36,37]$

$$
w_{\mathrm{Ag}} \frac{2 \pi}{\lambda_{0}} n_{\mathrm{GSP}}+\varphi=m \pi,
$$

where $w_{\mathrm{Ag}}$ as mentioned before denotes the width of the top nanograting array, $\lambda_{0}$ is the wavelength of the free space, $n_{\mathrm{GSP}}$ is the real part of the effective mode index, $\varphi$ is the additional phase acquired upon its reflection at the termination of the structure, and $m$ is an integer showing the order of the GSP mode. According to figure 2, the electric field distribution at the resonance wavelength of $\lambda_{1}$ demonstrates the excitation of fifth-order mode $(m=5)$ within the spacer layer, while the electric-field mode profiles at the 
(a)
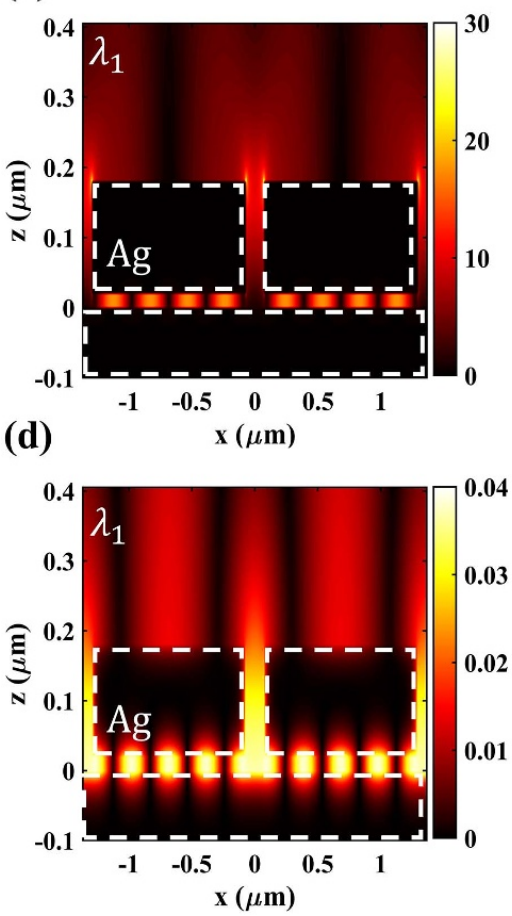

(b)
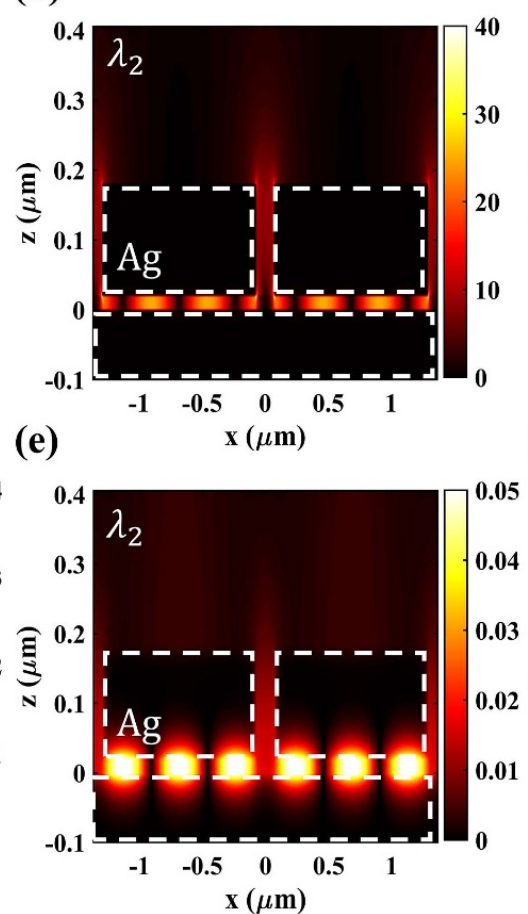

(c)
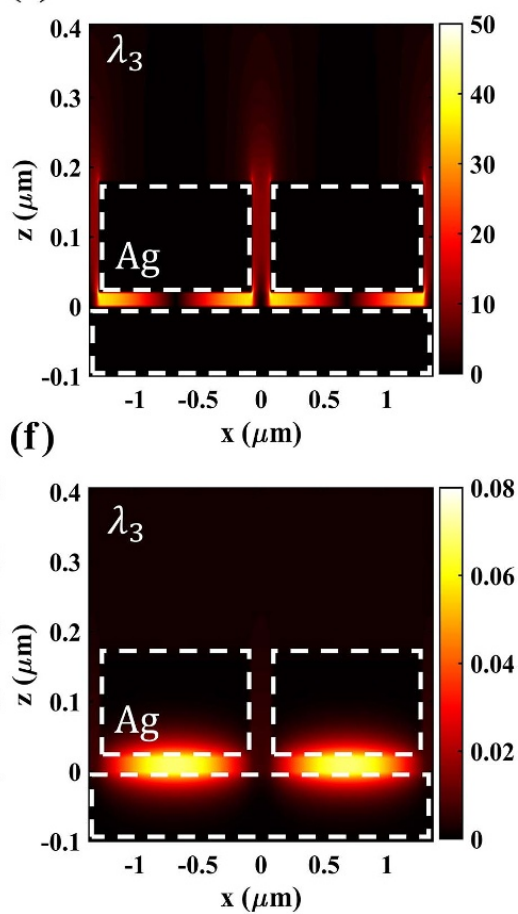

Figure 2. Absolute values of the total electric- and magnetic-field distributions on the $x-z$ plane obtained for a two-unit cell of design I at the resonance wavelengths of (a), (d) $\lambda_{1}=1524 \mathrm{~nm}$, (b), (e) $\lambda_{2}=2279 \mathrm{~nm}$, and (c), (f) $\lambda_{3}=6000 \mathrm{~nm}$, respectively. In all the figures, dashed white lines represent the Ag area.

resonance wavelengths of $\lambda_{2}$, and $\lambda_{3}$ correspond to the excitation of the third-order $(m=3)$ and first-order $(m=1)$ modes, respectively [34].

The calculated total magnetic-field distributions depicted in figures 2(d)-(f) also validate the type of the GSP-based resonances. In the fifth mode, anti-parallel currents in the top Ag nanograting and the bottom metallic layer create five anti-nodes of the magnetic fields inside the thin spacer layer. Accordingly, the third and first modes are due to the formation of three and first anti-nodes of the magnetic fields in the spacer layer, respectively. From the electric- and magneticfield distributions given in figures 2(a) and (d), it is observed that surface plasmons (SPs) are also excited between the air and the top Ag nanograting, and localized around the grating corners between the adjacent unit cells, creating an SP-induced light absorption at $\lambda_{1}$. Therefore, the origin of $\lambda_{1}$ is due to the formation of both SP and GSP resonances. While $\lambda_{2}$ and $\lambda_{3}$ are due to the excitations of GSP resonances. These facts can also be illustrated by calculating the absorbed-power densities given in figure 3. Figure 3(a) demonstrates that a small amount of absorption within the groove and the absorption between the top and bottom Ag layers are attributed to the combinations of both SP and GSP resonances at the resonance wavelength of $\lambda_{1}$. Furthermore, the absorbed power densities, given in figures 3(b) and (c), demonstrate that the most amount of absorption occurred between the top and bottom Ag layers (both interfaces of the dielectric layer), confirming the types of the resonance wavelengths of $\lambda_{2}$ and $\lambda_{3}$ are due to the excitation of GSPs.

\subsection{Geometric parameters effects}

The impact of changes of $t_{\mathrm{SiO}_{2}}, w_{\mathrm{Ag}}$, and $p$ on the overall absorption spectrum of design I is examined. Figure 3(d) represents the contour plot of absorptivity versus the wavelength for the different thicknesses of the spacer $\left(t_{\mathrm{SiO}_{2}}\right)$, while the other parameters of the structure are kept at their optimal values $\left(10 \mathrm{~nm} \leqslant t_{\mathrm{SiO}_{2}} \leqslant 200 \mathrm{~nm}\right.$ when $w_{\mathrm{Ag}}=1220 \mathrm{~nm}$, $t_{\mathrm{Ag}}=160 \mathrm{~nm}$ and $\left.p=1365 \mathrm{~nm}\right)$. The results illustrate triple narrowband perfect absorptions that are sensitive to the variation of the thickness of the spacer as it increases from $10 \mathrm{~nm}$ to $200 \mathrm{~nm}$. Increasing and getting far away from the optimized thickness value of the spacer (i.e. $t_{\mathrm{SiO}_{2}}=20 \mathrm{~nm}$, by considering the fact that the perfect absorption threshold is above 0.9) leads to a reduction in the amount of absorption, while the fourth resonance at longer wavelengths is efficiently excited by moving from a thinner to a thicker spacer. It is evident from figure $3(\mathrm{~d})$ that all the resonances are a function of the thickness of the spacer. In other words, by changing the thickness of $\mathrm{SiO}_{2}$ from $50 \mathrm{~nm}$ to $10 \mathrm{~nm}$, the resonance wavelengths are red-shifted toward the longer wavelength regions. This behavior of the resonances is in good agreement with the concept of GSP modes [38]. Therefore, according to the electric- and magnetic-field confinements within the spacer as shown in figures 2(a)-(f), all resonances more or less are affected by the thickness variation of the $\mathrm{SiO}_{2}$ layer. The normalized contour plot absorptivity response of the design I for different widths of the top Ag nanograting layer is shown in figure 3(e). The Ag width is swept from $1150 \mathrm{~nm}$ 
(a)


(b)
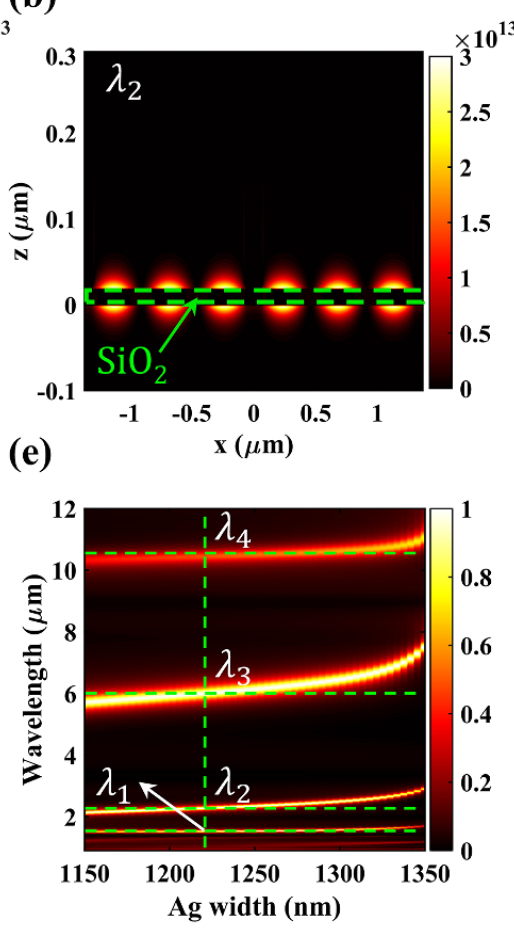

(c)
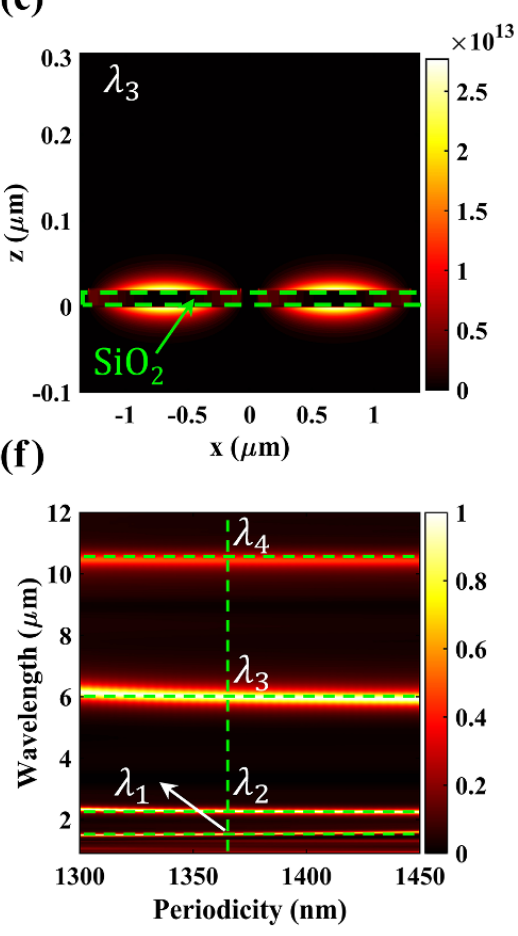

Figure 3. Absolute values of the absorbed-power densities on the $x-z$ plane obtained for a two-unit cell of design I at the resonance wavelengths of (a) $\lambda_{1}=1524 \mathrm{~nm}$, (b) $\lambda_{2}=2279 \mathrm{~nm}$, and (c) $\lambda_{3}=6000 \mathrm{~nm}$. In all the figures, dashed green lines represent the SiO 2 area. (d) Simulated contour plot of absorptivity as a function of wavelength for different thicknesses of the spacer $\left(t_{\mathrm{SiO}_{2}}\right)(10 \mathrm{~nm}$ $\leqslant t_{\mathrm{SiO}_{2}} \leqslant 200 \mathrm{~nm}$ when $t_{\mathrm{Ag}}=160 \mathrm{~nm}, w_{\mathrm{Ag}}=1220 \mathrm{~nm}$, and $p=1365 \mathrm{~nm}$ ). (h) Simulated contour plot of absorptivity versus wavelength for different widths $\left(w_{\mathrm{Ag}}\right)$ of the top Ag nanograting layer $\left(1150 \mathrm{~nm} \leqslant w_{\mathrm{Ag}} \leqslant 1350 \mathrm{~nm}\right.$ when $t_{\mathrm{SiO}_{2}}=20 \mathrm{~nm}, t_{\mathrm{Ag}}=160 \mathrm{~nm}$, and $\left.p=1365 \mathrm{~nm}\right)$ (f) Simulated contour plot of absorptivity versus wavelength for different periodicities $(p)$ of the structure $(1300 \mathrm{~nm} \leqslant p \leqslant 1450 \mathrm{~nm}$ when $t_{\mathrm{SiO}_{2}}=20 \mathrm{~nm}, t_{\mathrm{Ag}}=160 \mathrm{~nm}$, and $\left.w_{\mathrm{Ag}}=1220 \mathrm{~nm}\right)$. The interaction points of the dashed green lines correspond to the resonance wavelengths of $\lambda_{1}=1524 \mathrm{~nm}, \lambda_{2}=2279 \mathrm{~nm}, \lambda_{3}=6000 \mathrm{~nm}$, and $\lambda_{4}=10450 \mathrm{~nm}$.

to $1350 \mathrm{~nm}$, while the thickness of $\mathrm{SiO}_{2}$ is kept at its optimal value $\left(t_{\mathrm{SiO}_{2}}=20 \mathrm{~nm}\right)$. The resonance wavelengths of $\lambda_{2}$ and $\lambda_{3}$ are smoothly red-shifted by moving toward a wider grating. The red-shifted behavior of the resonances obtained by increasing the width of the top Ag nanograting are in complete agreement with the FP formula given in equation (1) [39]. As a result, it is almost possible to linearly tune the resonances of the nanoantenna emitter by changing the width of $\mathrm{Ag}$ nanograting. However, the resonance position of $\lambda_{1}$ is almost unaffected by increasing the width of the top $\mathrm{Ag}$ nanograting, confirming that the first resonance is excited by the combination of GSP and SP resonances. Figure 3(f) shows the variation of absorptivity versus wavelength at different values of the nanograting periodicity, while the other parameters are kept at their optimized values $(1300 \mathrm{~nm}$ $\leqslant p \leqslant 1450 \mathrm{~nm}$ when $t_{\mathrm{SiO}_{2}}=20 \mathrm{~nm}, w_{\mathrm{Ag}}=1220 \mathrm{~nm}$, and $\left.t_{\mathrm{Ag}}=160 \mathrm{~nm}\right)$. As expected, the periodicity does not affect the position of the GSP-based resonances as explained in $[40,41]$.

All in all, it is concluded that at specific dimensions $\left(20 \mathrm{~nm} / 1220 \mathrm{~nm} / 1365 \mathrm{~nm}\right.$ for $\left.t_{\mathrm{SiO}_{2}} / w_{\mathrm{Ag}} / p\right)$, the incident electromagnetic waves can dominantly couple to GSP modes which lead to a high amount of absorption at the resonance wavelengths of $\lambda_{1}, \lambda_{2}$, and $\lambda_{3}$.

\subsection{Power analysis}

To evaluate the thermal performance of the proposed nanoantenna emitter (design I), we calculate the spectral radiant emittance of a blackbody, a conventional surface, and the selective nanoantenna emitter at different temperatures. Planks' law which describes the spectral radiance emitted by a blackbody in thermal equilibrium is given by

$$
P(\lambda, T)=2 \pi h c^{2} / \lambda^{5}\left(\exp \left(\frac{h c}{\lambda k_{\mathrm{B}} T}\right)-1\right)^{-1}
$$

where $k_{\mathrm{B}}$ is Boltzmann's constant, $c$ is the speed of light in vacuum, and $\lambda$ is the wavelength of the incident light. The IR signatures of the conventional surface $\left[\operatorname{CS}(\lambda, T)=P(\lambda, T) \times\left(1-\varepsilon_{\text {atmosphere }}(\lambda, T)\right)\right]$ and the selective nanoantenna emitter $[\operatorname{NE}(\lambda, T)=P(\lambda, T) \times$ $\left.\left(1-\varepsilon_{\text {atmosphere }}(\lambda, T)\right) \times \varepsilon_{\text {structure }}(\lambda)\right]$ can be obtained from the data of the atmospheric absorption $\left[\varepsilon_{\text {atmosphere }}(\lambda, T)\right]$ and the calculated emissivity of design I $\left[\varepsilon_{\text {structure }}(\lambda)\right]$, respectively. Figure 4(a) compares the calculated IR signatures of the conventional surface and the selective nanoantenna emitter with the spectral radiant emittance of the blackbody radiation at $300 \mathrm{~K}$ by considering the radiation energy emitted 
(a)

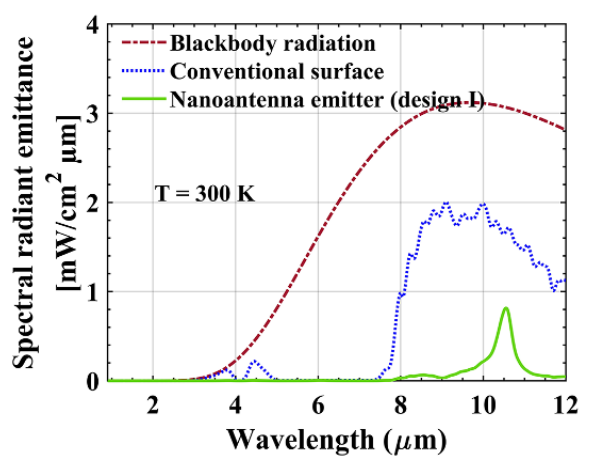

(b)

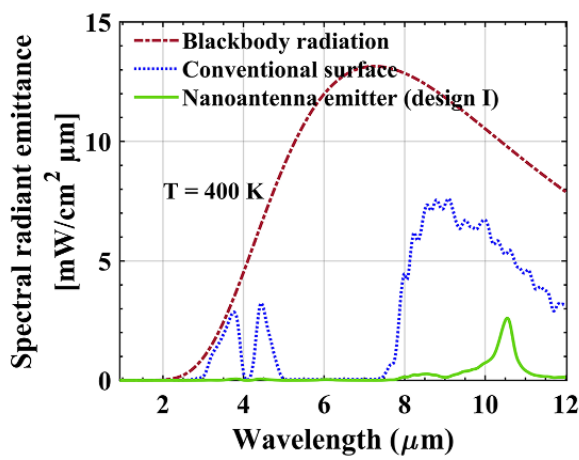

(c)

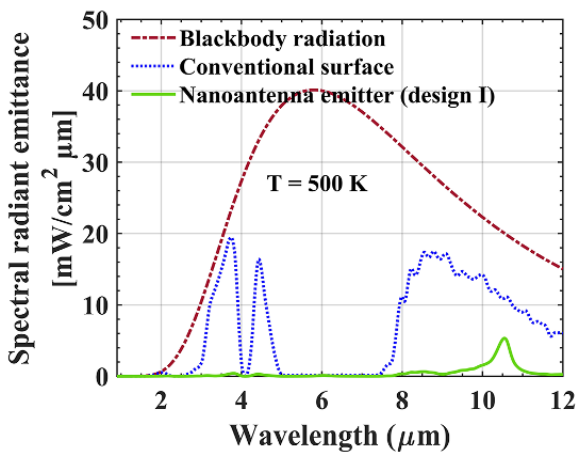

Figure 4. IR signatures of a blackbody, a conventional surface, and the selective nanoantenna emitter (design I) at different temperatures of (a) $300 \mathrm{~K}$, (b) $400 \mathrm{~K}$, and (c) $500 \mathrm{~K}$.

Table 1. The reduction rates of the selective nanoantenna emitter (design I).

\begin{tabular}{lccc}
\hline & $3-5 \mu \mathrm{m}$ & $8-12 \mu \mathrm{m}$ & $0.9-12 \mu \mathrm{m}$ \\
\hline$T=300 \mathrm{~K}$ & $99.71 \%$ & $94.76 \%$ & $96.09 \%$ \\
$T=400 \mathrm{~K}$ & $99.61 \%$ & $95.23 \%$ & $97.71 \%$ \\
$T=500 \mathrm{~K}$ & $99.53 \%$ & $95.55 \%$ & $98.68 \%$ \\
\hline
\end{tabular}

from the normal direction. The IR signatures of the selective nanoantenna emitter at the room temperature in the MWIR and LWIR ranges are reduced by $99.71 \%$, and $94.76 \%$ compared to the blackbody radiation, respectively. While the selective nanoantenna emitter can reduce the radiant energy by $96.09 \%$ throughout the entire wavelength band. As the temperature increases to $400 \mathrm{~K}$ and $500 \mathrm{~K}$, as shown in figures 4(b) and (c), the reduction rates of IR signatures for the selective nanoantenna emitter in the MWIR and LWIR ranges decrease but are still higher than $95 \%$. Notably, the reduction rate of the selective nanoantenna emitter is greater than $96 \%$ across a wide temperature varying from room temperature to $500 \mathrm{~K}$ for the entire wavelength range. Using the relation

$$
\text { Reduction rates }=\sqrt{\frac{\sum_{i=1}^{n}\left|P\left(\lambda_{i}, T\right)-N E\left(\lambda_{i}, T\right)\right|^{2}}{\sum_{i=1}^{n}\left|P\left(\lambda_{i}, T\right)\right|^{2}}},
$$

the reduction rates of the selective nanoantenna emitter (design I) in the wavelength ranges of $3-5 \mu \mathrm{m}, 8-12 \mu \mathrm{m}$, and the entire band for different temperatures are calculated and shown in table 1.

\section{Design II}

\subsection{Results and discussions}

In the next section of this study, we extend the functionality of design I to provide multifunctional operations including different thermal camouflage scenarios. The extended structure is called design II. The overall detection scenario of a hot object is schematically explained in figure 5(a). To hide from these surveillance devices, the surface of the object should be decorated with the proposed GSP-based nanoantenna emitters. Unlike MWIR and LWIR mode cameras that detect blackbody photons emitted from the object, SWIR mode cameras detect solar reflectance from the surface. Therefore, in an ideal scheme, the camouflage design should also perform as a broadband absorber in the 900-1700 $\mathrm{nm}$ range. To achieve a broadband absorption within the near-infrared (NIR) region and the main atmospheric absorption window, design I is modified with a highly lossy titanium (Ti) layer [42] embedded on an $\mathrm{SiO}_{2}$ layer and extended to design II with a multilayer grating as presented in figure 5(a). The thickness of each layer of grating is optimized and set to be $t_{\mathrm{Ag}}=70 \mathrm{~nm}$, $t^{\prime}{ }_{\mathrm{SiO}_{2}}=180 \mathrm{~nm}$, and $t_{\mathrm{Ti}}=5 \mathrm{~nm}$, respectively. The thickness of the insulator substrate which supports GSPs is considered as $t_{\mathrm{SiO}_{2}}=30 \mathrm{~nm}$. The unit cell is periodically arranged along the $x$ direction with $p=1200 \mathrm{~nm}$, and the corresponding unit cell width is $w=1160 \mathrm{~nm}$. Furthermore, the MIM grating structure in design II is separated by graphene layers from the substrate. The 2D graphene layers (single- or three-layer graphene) are added for the manipulation of the surface conductivity at the mid-infrared (MIR) region to have a real-time tunability in the infrared response of the structure within the atmospheric absorption window and quench the LWIR mode response, specifically the fourth resonance occurred in design II $\left(\lambda_{4}^{\prime}\right)$ due to intrinsic vibrational modes (optical phonons) of the $\mathrm{SiO}_{2}$ layer. The surface conductivity $\left(\sigma_{\mathrm{s}}\right)$ of a monolayer graphene in the simulations follows the well-established Kubo formula with intra-band $\left(\sigma_{\text {intra }}\right)$ and inter-band $\left(\sigma_{\text {inter }}\right)$ contributions as [43],

$$
\sigma_{\mathrm{s}}=\sigma_{\text {intra }}+\sigma_{\text {inter }}
$$

$$
\begin{gathered}
\sigma_{\text {intra }}=-j \frac{e^{2} k_{\mathrm{B}} T}{\pi \hbar^{2}(\omega-j 2 \Gamma)}\left[\frac{\mu_{c}}{k_{\mathrm{B}} T}+2 \ln \left(e^{-\mu_{c} / k_{\mathrm{B}} T}+1\right)\right], \\
\sigma_{\text {inter }} \simeq \frac{-j e^{2}}{4 \pi \hbar} \ln \left(\frac{2\left|\mu_{c}\right|-(\omega-j 2 \Gamma) \hbar}{2\left|\mu_{c}\right|+(\omega-j 2 \Gamma) \hbar}\right),
\end{gathered}
$$

where $\omega, T, e, \hbar$, and $k_{\mathrm{B}}$ are representing the angular-frequency of the incident plane wave, the temperature, electron charge, 

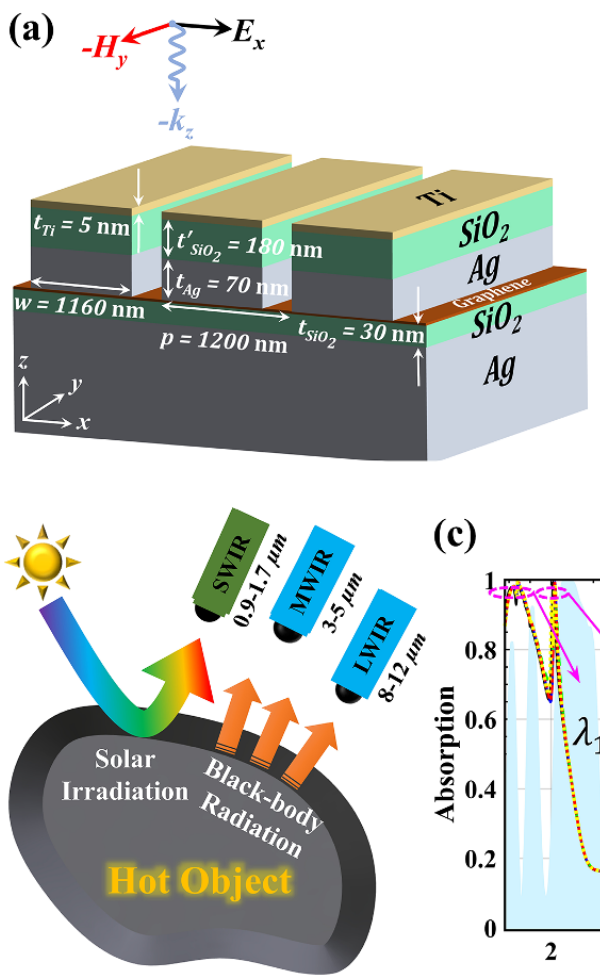

(e)

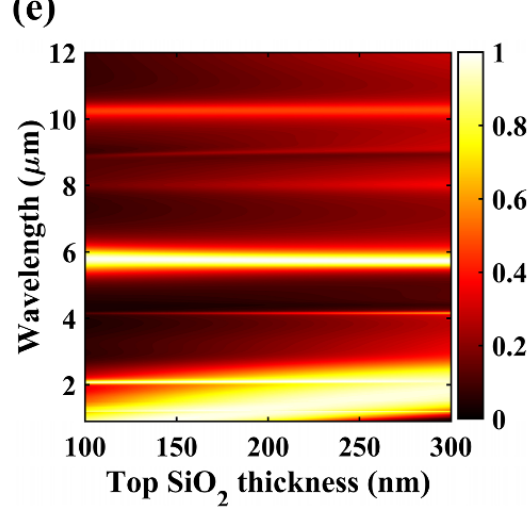

(b)



(d) (c)

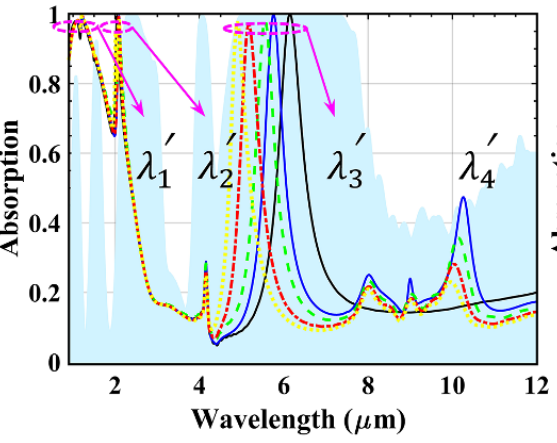

(f)


Figure 5. (a) Schematic diagram of the extended nanoantenna emitter (design II) and the overall detection scenario of a hot object. (b) Spectral absorption of design II without/with a single-layer $(N=1)$ graphene when $\mu_{c}=0.2 \mathrm{eV}, 0.4 \mathrm{eV}$, and $0.6 \mathrm{eV}$ at $T=300 \mathrm{~K}$ (c) Spectra absorption of design II without/with a three-layer $(N=3)$ graphene when $\mu_{c}=0.2 \mathrm{eV}, 0.4 \mathrm{eV}$, and $0.6 \mathrm{eV}$ at $T=300 \mathrm{~K}$. (d) The simulated absorption spectrum of design II with different values of scattering rate for the three-layer $(N=3)$ graphene case when $\mu_{c}=0.6 \mathrm{eV}$ at $T=300 \mathrm{~K}$. Simulated normalized contour plots of absorptivity versus wavelength for different thicknesses of (e) the top $\mathrm{SiO}_{2}$ layer $\left(100 \mathrm{~nm} \leqslant t^{\prime} \mathrm{SiO}_{2} \leqslant 300 \mathrm{~nm}\right.$ when $t_{\mathrm{Ag}}=70 \mathrm{~nm}, t_{\mathrm{Ti}}=5 \mathrm{~nm}, t_{\mathrm{SiO}_{2}}=30 \mathrm{~nm}, w=1160 \mathrm{~nm}$, and $\left.p=1200 \mathrm{~nm}\right)$, and (f) the thickness of Ti layer $\left(5 \mathrm{~nm} \leqslant t_{\mathrm{Ti}} \leqslant 25 \mathrm{~nm}\right.$ when $t_{\mathrm{Ag}}=70 \mathrm{~nm}, t^{\prime}{ }_{\mathrm{SiO}_{2}}=180 \mathrm{~nm}, t_{\mathrm{SiO}_{2}}=30 \mathrm{~nm}, w=1160 \mathrm{~nm}$, and $\left.p=1200 \mathrm{~nm}\right)$.

Planck's and Boltzmann's constants, respectively. In addition, $\mu_{c}$ and $2 \Gamma\left(2 \Gamma=\frac{\hbar}{\tau}, \tau\right.$ is the electron-phonon relaxation time $)$ are the chemical potential and scattering rate of a monolayer graphene accounting for the Fermi energy $\left(E_{\mathrm{F}}\right)$ level and the intrinsic losses, respectively. One of the main advantages of using graphene is that its Fermi level can be easily tuned over a wide range by applying an external gate voltage (electrostatic biasing), leading to different surface conductivities. Therefore, it is possible to tune the MIR resonance of design II and reduce the peak absorption of the fourth resonance by varying the Fermi level via electrostatic biasing. The relation between Fermi energy level and the electrostatic biasing is given by an approximate closed-form expression as [44]

$$
E_{\mathrm{F}}=\hbar v_{\mathrm{F}} \sqrt{\frac{\pi C_{d} V_{\mathrm{g}}}{e}},
$$

where $C_{d}=\varepsilon_{d} \varepsilon_{0} / t_{s}$ is the electrostatic gate capacitance, $t_{s}$ is the thickness of gate oxide, $V_{\mathrm{g}}$ is the applied gate voltage, and $v_{\mathrm{F}}$ is the Fermi velocity $\left(1.0 \times 10^{6} \mathrm{~m} \mathrm{~s}^{-1}\right.$ in graphene), respectively. As a result, the electrostatic biasing of the graphene layers in design II can be achieved by placing conductive contacts between the graphene and the bottom Ag reflector (electrostatic ground) and applying a gate voltage. The bias then allows for controlling the chemical potential of graphene layers independently $[44,45]$. 
Table 2. Absorption resonance wavelengths of the proposed wavelength-selective nanoantenna emitter (design II) without a graphene layer, with a single-layer graphene, and a three-layer graphene (at different chemical potentials: $\mu_{c}=0.2 \mathrm{eV}, 0.4 \mathrm{eV}$, and $0.6 \mathrm{eV}$ at $T=300 \mathrm{~K}$ ), together with peak values of the fourth resonance and the corresponding relative reduction rates obtained via comparing the results with design II without a graphene layer.

\begin{tabular}{|c|c|c|c|c|c|c|c|}
\hline & & \multicolumn{4}{|c|}{ Resonance } & \multirow{2}{*}{\multicolumn{2}{|c|}{$\begin{array}{c}\text { Intensity of \#4 } \\
\text { and reduction rates }\end{array}$}} \\
\hline & & $\# 1$ & $\# 2$ & \#3 & $\# 4$ & & \\
\hline Design II (with a & $\boldsymbol{\mu}_{c}=0.2 \mathrm{eV}$ & $\lambda_{1}^{\prime}=929-1472 \mathrm{~nm}$ & $\lambda_{2}^{\prime}=2080 \mathrm{~nm}$ & $\lambda_{3}^{\prime}=5656 \mathrm{~nm}$ & $\lambda_{4}^{\prime}=10234 \mathrm{~nm}$ & 0.43 & $-8.51 \%$ \\
\hline $\begin{array}{l}\text { Design II (with a } \\
\text { single-layer graphene) }\end{array}$ & $\mu_{c}=0.4 \mathrm{eV}$ & $\lambda_{1}^{\prime}=929-1472 \mathrm{~nm}$ & $\lambda_{2}^{\prime}=2080 \mathrm{~nm}$ & $\lambda_{3}^{\prime}=5551 \mathrm{~nm}$ & $\lambda_{4}^{\prime}=10184 \mathrm{~nm}$ & 0.39 & $-17.02 \%$ \\
\hline \multirow{3}{*}{$\begin{array}{l}\text { Design II (with a } \\
\text { three-layer graphene) }\end{array}$} & $\boldsymbol{\mu}_{\boldsymbol{c}}=0.2 \mathrm{eV}$ & $\lambda_{1}^{\prime}=929-1472 \mathrm{~nm}$ & $\lambda_{2}^{\prime}=2080 \mathrm{~nm}$ & $\lambda_{3}^{\prime}=5555 \mathrm{~nm}$ & $\lambda_{4}^{\prime}=10135 \mathrm{~nm}$ & 0.35 & $-25.53 \%$ \\
\hline & $\boldsymbol{\mu}_{\boldsymbol{c}}=0.4 \mathrm{eV}$ & $\lambda_{1}^{\prime}=929-1472 \mathrm{~nm}$ & $\lambda_{2}^{\prime}=2080 \mathrm{~nm}$ & $\lambda_{3}^{\prime}=5170 \mathrm{~nm}$ & $\lambda_{4}^{\prime}=10032 \mathrm{~nm}$ & 0.28 & $-40.42 \%$ \\
\hline & $\mu_{c}=0.6 \mathrm{eV}$ & $\lambda_{1}^{\prime}=929-1472 \mathrm{~nm}$ & $\lambda_{2}^{\prime}=2080 \mathrm{~nm}$ & $\lambda_{3}^{\prime}=4896 \mathrm{~nm}$ & $\lambda_{4}^{\prime}=9957 \mathrm{~nm}$ & 0.22 & $-53.19 \%$ \\
\hline
\end{tabular}

The simulated absorption spectra of design II including different cases are presented in figure 5(b) when $\Gamma=$ $0.0032 \mathrm{eV}$ and $T=300 \mathrm{~K}$. The extension of design I to design II without a graphene layer leads to having a broadband resonance $\left(\lambda_{1}^{\prime}\right)$ with a perfect absorption ranging from $929 \mathrm{~nm}$ to $1472 \mathrm{~nm}$ (suitable for solar applications [46, 47]), where three narrowband absorptions at the longer wavelengths of $\lambda_{2}^{\prime}=$ $2080 \mathrm{~nm}, \lambda_{3}^{\prime}=5750 \mathrm{~nm}$, and $\lambda_{4}^{\prime}=10260 \mathrm{~nm}$ are excited (see figure 5(b)). According to the monolayer graphene characteristic [48, 49], the obtained broadband response of design II should stay almost unchanged in the NIR region, while the $\lambda_{3}^{\prime}$ and $\lambda_{4}^{\prime}$ resonances at the MIR region experience blue-shifts by adding the single-layer graphene and increasing $\mu_{c}$ from $0.2 \mathrm{eV}$ to $0.6 \mathrm{eV}$. In addition, the amount of absorption at the $\lambda_{4}^{\prime}$ resonance, caused by the vibrational modes of $\mathrm{SiO}_{2}$ layer, decreases by $25.53 \%$ (see the trend between the blue and yellow dot lines in figure 5(b)) for those cases without the graphene layer and with the single-layer graphene, when $\mu_{c}$ is $0.6 \mathrm{eV}$. This is due to the fact that a partial amount of power is reflected back from the single-layer graphene and the localization of the electric field decreases within the lower $\mathrm{SiO}_{2}$ layer. As several works have been reported to effectively design few-layer graphene structures for tuning the resonances at the MIR region [50,51], we next demonstrate the optical performance of design II for the case of the three-layer $(N=3)$ graphene. Here, it is taken into consideration that the surface conductivity $\left(\sigma_{\mathrm{s}}\right)$ for $N$-layer graphene is defined as $N \sigma_{\text {s }}$ [52]. Figure 5(c) shows the resonance wavelengths of the three-layer graphene device obtained from FDTD method as a function of $\mu_{c}$ ranging from $0.2 \mathrm{eV}$ to $0.6 \mathrm{eV}$. The resonance wavelengths exhibit a similar trend as observed in the single-layer structure in terms of blue-shifting of the MIR resonances and reducing the peak absorption of the fourth resonance. It is observed in figure 5(c) that increasing the number of graphene layers from $N=1$ to $N=3$ can further shift the MIR resonance wavelengths toward the shorter wavelength regions. In addition, compared to the case without the graphene layer, the structure containing the three-layer graphene with $\mu_{c}=$ $0.6 \mathrm{eV}$, can suppress the $\lambda_{4}^{\prime}$ absorption peak by $53.19 \%$ (see the trend between the blue and yellow dot lines in figure 5(c)).
The three-layer graphene device also suppresses the $\lambda_{4}^{\prime}$ absorption peak $\sim 2$.1 times more than the one obtained by the singlelayer graphene structure. It should be noted that the general functionality of design II with graphene layers is negligibly affected for different values of scattering rate as demonstrated for the three-layer graphene device in figure 5(d) when the chemical potential is assumed $0.6 \mathrm{eV}$. This means that variation of scattering rate due to the impurities, defects, and interfaces for realistic graphene layers $[53,54]$ does not affect the response of the proposed structure. All in all, the absorption spectra of design II perfectly match the atmospheric absorption windows with tunability characteristic [55] that make it suitable for different thermal camouflage scenarios as well as solar energy harvesting application due to the broadband absorption within a wide spectral range of the NIR region. The absorption resonance wavelengths of design II, together with the peak values of the fourth resonance and the corresponding relative reduction rates are all summarized in table 2 .

\subsection{Geometric parameters effects}

In order to investigate the performance of each layer and their contributions to the resonances, we once again examine how changes in $t^{\prime}{ }_{\mathrm{SiO}_{2}}$, and $t_{\mathrm{Ti}}$ can affect the overall absorption spectrum of design II without considering the graphene layer. Figure 5(e) shows the dependence of the normalized absorbed power by the structure on the changes of $t^{\prime} \mathrm{SiO}_{2}$, while keeping all of the other geometrical parameters unchanged $(100 \mathrm{~nm} \leqslant$ $t^{\prime}{ }_{\mathrm{SiO}_{2}} \leqslant 300 \mathrm{~nm}$ when $t_{\mathrm{Ag}}=70 \mathrm{~nm}, t_{\mathrm{Ti}}=5 \mathrm{~nm}, t_{\mathrm{SiO}_{2}}=30 \mathrm{~nm}$, $w=1160 \mathrm{~nm}$ and $p=1200 \mathrm{~nm}$ ). The result exhibits a broadband perfect absorption at the NIR region (with a peak absorption at $\left(\lambda_{1}^{\prime}\right)$ ) and a sharp narrowband response $\left(\lambda_{3}^{\prime}\right)$ at the atmospheric absorption window for all $t_{\mathrm{SiO}_{2}}^{\prime}$ values, where only the former resonance experiences a redshift as the spacer thickness increases. This phenomenon demonstrates that the NIR response $\left(\lambda_{1}^{\prime}\right)$ is due to the added top layers to the system, while the wavelength resonances including $\lambda_{2}^{\prime}$ and $\lambda_{3}^{\prime}$ are due to the formation of gap plasmons (similar to design I) in the interfaces of the $\mathrm{SiO} 2$ substrate and the Ag layers. To have a solar absorber operating in the NIR region 
(a)



(d)

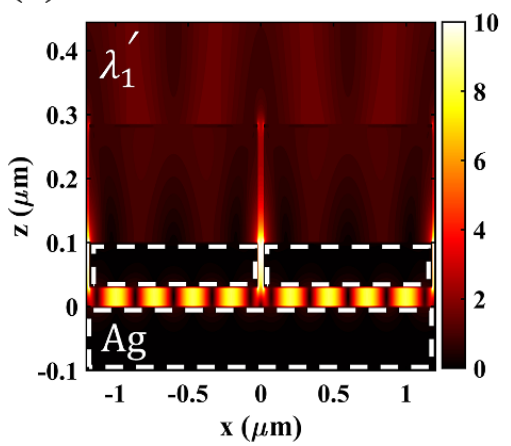

(b)

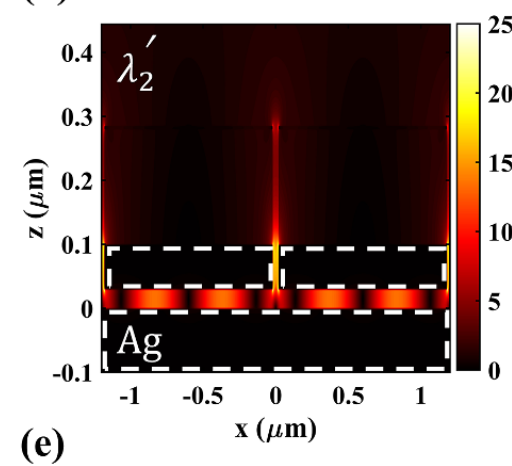

(e)

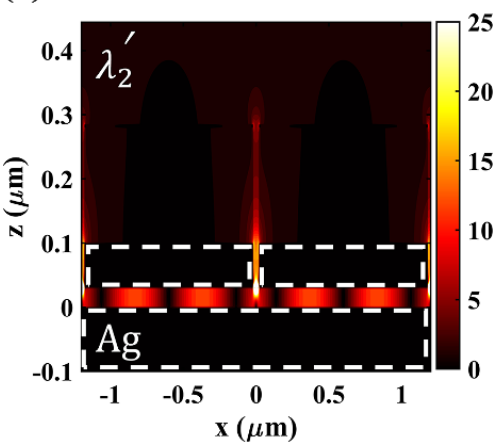

(c)

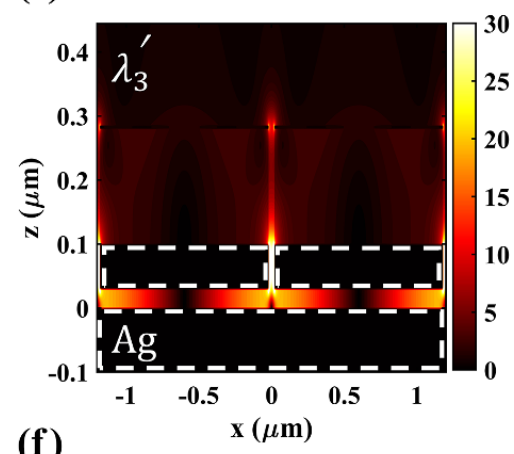

(f)

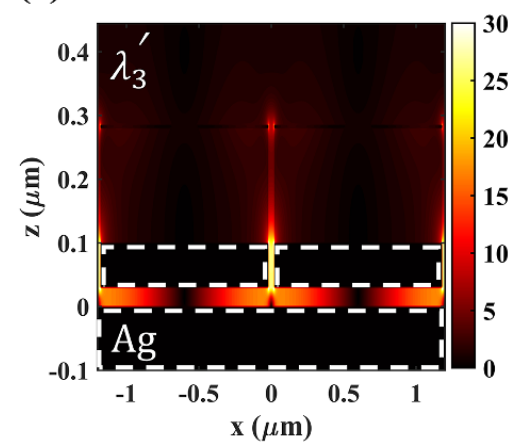

Figure 6. Absolute values of the total electric-field distributions on the $x-z$ plane obtained for a two-unit cell of design II (without graphene layers) at the resonance wavelengths of (a) $\lambda_{1}^{\prime}=1205 \mathrm{~nm}$, (b) $\lambda_{2}^{\prime}=2080 \mathrm{~nm}$, and (c) $\lambda_{3}^{\prime}=5750 \mathrm{~nm}$, respectively. Absolute values of the total electric-field distributions on the $x-z$ plane obtained for a two-unit cell of design II (with the three-layer graphene when $\mu_{c}=0.6 \mathrm{eV}$ ) at the resonance wavelengths of (d) $\lambda_{4}^{\prime}=1205 \mathrm{~nm}$, (e) $\lambda_{4}^{\prime}=2080 \mathrm{~nm}$, and (f) $\lambda_{4}^{\prime}=4896 \mathrm{~nm}$, respectively. In all the figures, dashed white lines represent the Ag area.

with an absorption above $90 \%$, the optimal thickness of the $\mathrm{SiO}_{2}$ interlayer $\left(t^{\prime} \mathrm{SiO}_{2}\right)$ is set at $180 \mathrm{~nm}$. Figure 5(f) shows the impact of changes in $t_{\mathrm{Ti}}$ on the normalized spectral absorption of design II without considering graphene layer, when it varies from $5 \mathrm{~nm}$ to $25 \mathrm{~nm}\left(5 \mathrm{~nm} \leqslant t_{\mathrm{Ti}} \leqslant 25 \mathrm{~nm}\right.$ when $t_{\mathrm{Ag}}=70 \mathrm{~nm}, t^{\prime}{ }_{\mathrm{SiO}_{2}}=180 \mathrm{~nm}, t_{\mathrm{SiO}_{2}}=30 \mathrm{~nm}, w=1160 \mathrm{~nm}$ and $p=1200 \mathrm{~nm}$ ). Accordingly, the resonances around $8 \mu \mathrm{m}$ and $10 \mu \mathrm{m}$ are efficiently excited by moving toward thicker values of the Ti layer $\left(t_{\mathrm{Ti}}>20 \mathrm{~nm}\right)$ besides the reduction in the amount of absorption of the resonance $\left(\lambda_{3}^{\prime}\right)$ in the atmospheric absorption window. As a result, the optimal performance can be achieved with the thickness of $t_{T i}=5 \mathrm{~nm}$.

\subsection{Physical mechanism}

In order to reveal the physical mechanism of the proposed selective nanoantenna emitter (design II) under the normal incidence, electric-field distributions at the resonance wavelengths are obtained on the $\mathrm{x}-z$ plane and illustrated in figure 6 , corresponding to the cases without graphene and with the three-layer graphene $\left(\mu_{c}=0.6 \mathrm{eV}\right)$. It is seen from figures 6(a) and (d) that at the resonance wavelength of $\lambda_{1}^{\prime}=$ $1205 \mathrm{~nm}$, the incident light penetrates thorugh the thin Ti layer and bounces back at the bottom Ag grating layer. The two reflectors dominantly excite the FP cavity resonance leading to a broaden absorption response thanks to the lossy behavior of Ti in the NIR region [42]. However, the distributions of the electric-field at the other excited resonances are intrinsically different. In particular, at the resonance wavelength of $\lambda_{2}^{\prime}=$ $2080 \mathrm{~nm}$ presented in figures 6(b) and (e), the electric-field localizations inside the spacer layer correspond to the excitation of the third-order mode GSP resonance [34-39], while the absorptions at the resonance wavelengths of $\lambda_{3}^{\prime}=5750 \mathrm{~nm}$ and $\lambda_{3}^{\prime}=4986 \mathrm{~nm}$ are dominantly attributed to the excitation of GSPs with the first-order mode. This can be seen in figures 6(c) and (f) for the design without graphene layer and the design with the three-layer graphene, respectively.

\subsection{Power analysis}

To evaluate the performances of the second nanoantenna emitter (design II) in thermal camouflage technology, we once again calculate the IR signatures of the conventional surface and the extended nanoantenna emitter (design II without/with the three-layer graphene when $\mu_{c}=0.6 \mathrm{eV}$ ) to compare with the spectral radiant emittance of the blackbody radiation at $300 \mathrm{~K}$. The IR signatures in the MWIR and LWIR wavelength ranges can be reduced by $98.67 \%$ and $88.74 \%$ without considering the graphene layers as shown in figure 7(a). As the temperature increases, these two values become $97.90 \%$ and $88.64 \%$ at $400 \mathrm{~K}$ and $96.92 \%$ and $88.65 \%$ at $500 \mathrm{~K}$ as illustrated in figures 7(b) and (c). Meanwhile, the selective nanoantenna emitter can reduce the radiant energy by $91.50 \%$, $94.40 \%$, and $96.21 \%$ for the whole wavelength spectrum at 
(a)

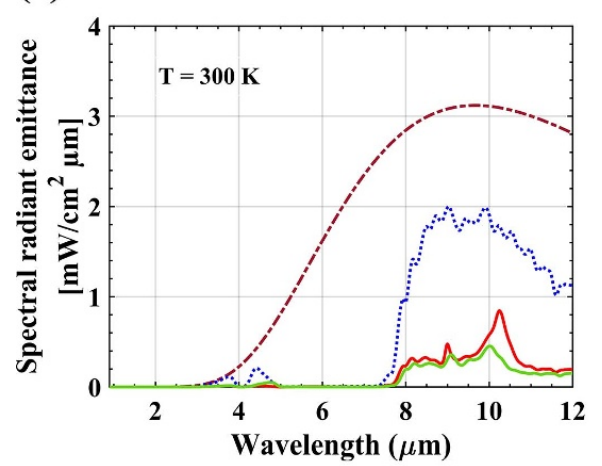

(b)

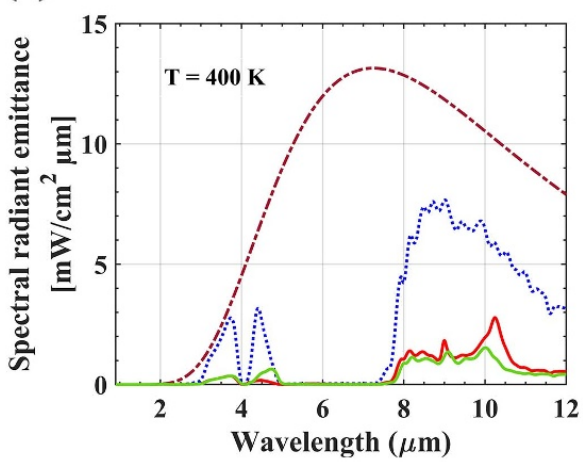

(c)

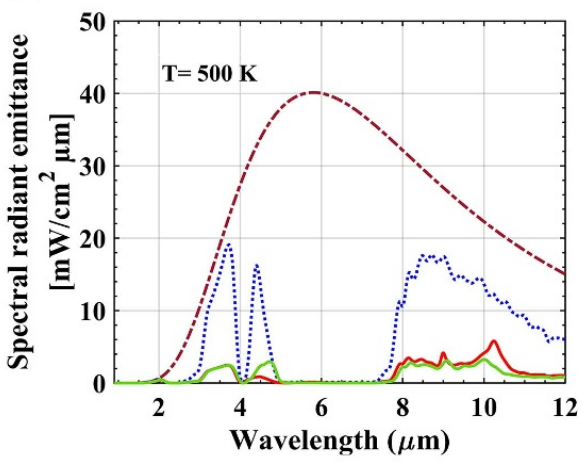

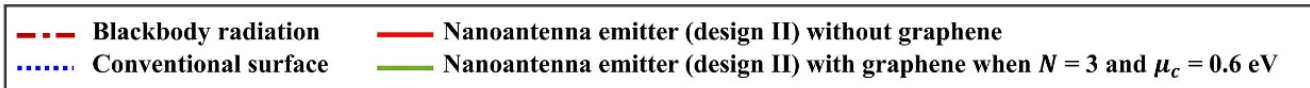

Figure 7. IR signatures of a blackbody, a conventional surface, and the selective nanoantenna emitter (design II without/with the three-layer graphene $(N=3)$ when $\mu_{c}=0.6 \mathrm{eV}$ ) at different temperatures of (a) $300 \mathrm{~K}$, (b) $400 \mathrm{~K}$, and (c) $500 \mathrm{~K}$.

Table 3. The reduction rates of the selective nanoantenna emitter (design II without/with the single- and three-layer graphene when $\mu_{c}=0.2 \mathrm{eV}, 0.4 \mathrm{eV}$, and $0.6 \mathrm{eV}$ ) in the wavelength ranges of $3-5 \mu \mathrm{m}, 8-12 \mu \mathrm{m}$, and the entire band at different temperatures.

\begin{tabular}{|c|c|c|c|c|c|}
\hline & & \multicolumn{4}{|c|}{ The reduction rates } \\
\hline & & Temperatures & $3-5 \mu \mathrm{m}$ & $8-12 \mu \mathrm{m}$ & $0.9-12 \mu \mathrm{m}$ \\
\hline \multirow{3}{*}{ Design II (without a graphene layer) } & & $300 \mathrm{~K}$ & $98.67 \%$ & $88.74 \%$ & $91.50 \%$ \\
\hline & & $400 \mathrm{~K}$ & $97.90 \%$ & $88.64 \%$ & $94.40 \%$ \\
\hline & & $500 \mathrm{~K}$ & $96.92 \%$ & $88.65 \%$ & $96.21 \%$ \\
\hline \multirow[t]{8}{*}{ Design II (with a single-layer graphene) } & $\boldsymbol{\mu}_{c}=0.2 \mathrm{eV}$ & $300 \mathrm{~K}$ & $98.56 \%$ & $89.21 \%$ & $91.85 \%$ \\
\hline & & $500 \mathrm{~K}$ & $96.82 \%$ & $89.06 \%$ & $96.29 \%$ \\
\hline & $\boldsymbol{\mu}_{c}=0.4 \mathrm{eV}$ & $300 \mathrm{~K}$ & $98.53 \%$ & $89.76 \%$ & $92.25 \%$ \\
\hline & & $400 \mathrm{~K}$ & $97.76 \%$ & $89.57 \%$ & $94.82 \%$ \\
\hline & & $500 \mathrm{~K}$ & $96.79 \%$ & $89.53 \%$ & $96.41 \%$ \\
\hline & $\boldsymbol{\mu}_{c}=0.6 \mathrm{eV}$ & $300 \mathrm{~K}$ & $98.41 \%$ & $90.22 \%$ & $92.59 \%$ \\
\hline & & $400 \mathrm{~K}$ & $97.67 \%$ & $90.00 \%$ & $95.01 \%$ \\
\hline & & $500 \mathrm{~K}$ & $96.74 \%$ & $89.94 \%$ & $96.51 \%$ \\
\hline & & $400 \mathrm{~K}$ & $97.53 \%$ & $89.87 \%$ & $94.94 \%$ \\
\hline & & $500 \mathrm{~K}$ & $96.57 \%$ & $89.81 \%$ & $96.44 \%$ \\
\hline & $\boldsymbol{\mu}_{c}=0.4 \mathrm{eV}$ & $300 \mathrm{~K}$ & $97.74 \%$ & $91.29 \%$ & $93.37 \%$ \\
\hline & & $400 \mathrm{~K}$ & $97.10 \%$ & $91.00 \%$ & $95.43 \%$ \\
\hline & & $500 \mathrm{~K}$ & $96.28 \%$ & $90.89 \%$ & $96.67 \%$ \\
\hline & $\mu_{c}=0.6 \mathrm{eV}$ & $300 \mathrm{~K}$ & $95.31 \%$ & $92.10 \%$ & $93.96 \%$ \\
\hline & & $400 \mathrm{~K}$ & $95.00 \%$ & $91.79 \%$ & $95.67 \%$ \\
\hline & & $500 \mathrm{~K}$ & $94.57 \%$ & $91.65 \%$ & $96.56 \%$ \\
\hline
\end{tabular}

$300 \mathrm{~K}, 400 \mathrm{~K}$, and $500 \mathrm{~K}$, respectively. Figures 7(a)-(c) also show the IR signatures of the selective nanoantenna emitter with the three-layer graphene when $\mu_{c}=0.6 \mathrm{eV}$. The results show better performance in terms of the reduction rate in comparison to the case without graphene layers. Table 3 summarizes the reduction rates of the selective nanoantenna emitter (design II) for all cases in the wavelength ranges 3-5 $\mu \mathrm{m}$, $8-12 \mu \mathrm{m}$, and the entire band at different temperatures. In general, the reduction rates exhibit a similar trend for all cases such that the IR signatures decrease in the MWIR and LWIR ranges as the temperature increases, while the reduction rate for the entire wavelength range increases as the temperature increases.

\section{Conclusions}

In conclusion, we proposed a GSP-based wavelength-selective nanoantenna emitter (design I) suitable for multiple thermal camouflage scenarios. Triple narrowband perfect absorptions are achieved at the resonance wavelengths of $1524 \mathrm{~nm}$, $2279 \mathrm{~nm}$, and $6000 \mathrm{~nm}$, whose absorption peaks perfectly match the atmospheric absorption spectrum. The proposed nanoantenna emitter is designed to have low emissivity within atmospheric windows and its performance is validated using numerical and analytical methods. Later, we proposed a multilayer grating structure as an extension of design I to cover solar thermal application besides multiple thermal camouflage 
scenarios. Design II contains a nearly perfect absorption ranging from $929 \mathrm{~nm}$ to $1472 \mathrm{~nm}$ and a narrowband absorption at the resonance wavelength of $5750 \mathrm{~nm}$, where the later absorption resonance is tuned and modified by adding graphene layers. Furthermore, we have also demonstrated that the multilayer graphene structure could outperform the single-layer one in terms of shifting the MIR resonance wavelength and reducing the absorption peak of the fourth response. Finally, the performances of the selective nanoantenna emitters in thermal camouflage applications are validated by calculating the spectral radiant emittances. It is shown that the infrared signatures of design I and design II (without/with graphene layers) are significantly reduced compared to the blackbody radiation energy for the entire wavelength spectrum at least by $96 \%$ and $91 \%$, respectively, ranging from the room temperature to $500 \mathrm{~K}$.

\section{Data availability statement}

All data that support the findings of this study are included within the article (and any supplementary files).

\section{Acknowledgments}

E Ozbay acknowledges partial support from the Turkish Academy of Sciences (TUBA).

\section{ORCID iDs}

Ataollah Kalantari Osgouei (D) https://orcid.org/0000-00020971-7687

Bahram Khalichi (D) https://orcid.org/0000-0002-9465-1044

\section{References}

[1] Zhao D, Aili A, Zhai Y, Xu S, Tan G, Yin X and Yang R 2019 Radiative sky cooling: fundamental principles, materials, and applications Appl. Phys. Rev. 6021306

[2] Sun X, Sun Y, Zhou Z, Alam M A and Bermel P 2017 Radiative sky cooling: fundamental physics, materials, structures, and applications Nanophotonics 6 997-1015

[3] Liu D, Ji H, Peng R, Cheng H and Zhang C 2018 Infrared chameleon-like behavior from $\mathrm{VO}_{2}(\mathrm{M})$ thin films prepared by transformation of metastable $\mathrm{VO}_{2}(\mathrm{~B})$ for adaptive camouflage in both thermal atmospheric windows Solar Energy Mater. Solar Cells 185 210-7

[4] Kong A, Cai B, Shi P and Yuan X C 2019 Ultra-broadband all-dielectric metamaterial thermal emitter for passive radiative cooling Opt. Express 27 30102-15

[5] Ko B, Lee D, Badloe T and Rho J 2019 Metamaterial-based radiative cooling: towards energy-free all-day cooling Energies 1289

[6] Zhou J, Liu Z, Liu G, Pan P, Liu X, Tang C and Wang J 2020 Ultra-broadband solar absorbers for high-efficiency thermophotovoltaics Opt. Express 28 36476-86

[7] Maremi F T, Lee N, Choi G, Kim T and Cho H H 2018 Design of multilayer ring emitter based on metamaterial for thermophotovoltaic applications Energies 112299

[8] Osgouei A K, Hajian H, Khalichi B, Serebryannikov A E, Ghobadi A and Ozbay E 2021 Active tuning from narrowband to broadband absorbers using a sub-wavelength $\mathrm{VO}_{2}$ embedded layer Plasmonics 16 1-9

[9] Hedayati M K, Javaherirahim M, Mozooni B, Abdelaziz R, Tavassolizadeh A, Chakravadhanula V S K, Zaporojtchenko V, Strunkus T, Faupel F and Elbahri M 2011 Design of a perfect black absorber at visible frequencies using plasmonic metamaterials Adv. Mater. $235410-4$

[10] Khalichi B, Ghobadi A, Osgouei A K and Ozbay E 2021 Diodelike high-contrast asymmetric transmission of linearly polarized waves based on plasmon-tunneling effect coupling to electromagnetic radiation modes J. Appl. Phys. 54365102

[11] Baranov D G, Xiao Y, Nechepurenko I A, Krasnok A, Alù A and Kats M A 2019 Nanophotonic engineering of far-field thermal emitters Nat. Mater. 18 920-30

[12] Lou F, Zhao R, He J, Jia Z, Su X, Wang Z, Hou J and Zhang B 2015 Nanosecond-pulsed, dual-wavelength, passively Q-switched ytterbium-doped bulk laser based on few-layer $\mathrm{MoS}_{2}$ saturable absorber Photonics Res. 3 A $25-\mathrm{A} 29$

[13] Kim T, Bae J-Y, Lee N and Cho H H 2019 Hierarchical metamaterials for multispectral camouflage of infrared and microwaves Adv. Funct. Mater. 291807319

[14] Kim J, Han K and Hahn J W 2017 Selective dual-band metamaterial perfect absorber for infrared stealth technology Sci. Rep. 7 1-9

[15] Salihoglu O, Uzlu H B, Yakar O, Aas S, Balci O, Kakenov N, Balci S, Olcum S, Süzer S and Kocabas C 2018 Graphene-based adaptive thermal camouflage Nano Lett. 18 4541-8

[16] Zhu H, Li Q, Zheng C, Hong Y, Xu Z, Wang H, Shen W, Kaur S, Ghosh P and Qiu M 2020 High-temperature infrared camouflage with efficient thermal management Light: Sci. Appl. 9 1-8

[17] Peng L, Liu D, Cheng H, Zhou S and Zu M 2018 A multilayer film based selective thermal emitter for infrared stealth technology Adv. Opt. Mater. 61801006

[18] Buhara E, Ghobadi A, Khalichi B, Kocer H and Ozbay E 2021 Mid-infrared adaptive thermal camouflage using a phase-change material coupled dielectric nanoantenna $J$. Appl. Phys. $\mathbf{5 4} 265105$

[19] Ghobadi A, Hajian H, Gokbayrak M, Dereshgi S A, Toprak A, Butun B and Ozbay E 2017 Visible light nearly perfect absorber: an optimum unit cell arrangement for near absolute polarization insensitivity $O p t$. Express 25 27624-34

[20] Li Z, Stan L, Czaplewski D A, Yang X and Gao J 2018 Wavelength-selective mid-infrared metamaterial absorbers with multiple tungsten cross resonators Opt. Express 26 5616-31

[21] Liu X, Tyler T, Starr T, Starr A F, Jokerst N M and Padilla W J 2011 Taming the blackbody with infrared metamaterials as selective thermal emitters Phys. Rev. Lett. 107045901

[22] Ha D T, Dzung D N, Van Ngoc N, Tung B S, Pham T S, Lee Y, Chen L Y, Xuan Khuyen B and Dinh Lam V 2021 Switching between perfect absorption and polarization conversion, based on hybrid metamaterial in the $\mathrm{GHz}$ and THz bands J. Appl. Phys. $\mathbf{5 4} 234003$

[23] Osgouei A K, Hajian H, Serebryannikov A E and Ozbay E 2021 Hybrid indium tin oxide-Au metamaterial as a multiband bi-functional light absorber in the visible and near-infrared ranges J. Appl. Phys. 54275102

[24] Lee N, Kim T, Lim J-S, Chang I and Cho H H 2019 Metamaterial-selective emitter for maximizing infrared camouflage performance with energy dissipation ACS Appl. Mater. Interfaces 11 21250-7 
[25] Kang Q, Li D, Guo K, Gao J and Guo Z 2021 Tunable thermal camouflage based on GST plasmonic metamaterial Nanomaterials 11260

[26] Li M, Liu D, Cheng H, Peng L and Zu M 2020 Manipulating metals for adaptive thermal camouflage $S c i$. $A d v$. 6 eaba3494

[27] Feng X, Xie X, Pu M, Ma X, Guo Y, Li X and Luo X 2020 Hierarchical metamaterials for laser-infrared-microwave compatible camouflage Opt. Express 28 9445-53

[28] Moharam M G, Pommet D A, Grann E B and Gaylord T K 1995 Stable implementation of the rigorous coupled-wave analysis for surface-relief gratings: enhanced transmittance matrix approach J. Opt. Soc. Am. A 12 1077-86

[29] Lumerical F 2018 Solutions, inc

[30] Lide D R (ed) 2004 CRC Handbook of Chemistry and Physics vol 85 (Boca Raton, FL: CRC Press)

[31] Palik E D 1998 Handbook of Optical Constants of Solids vol 3 (New York: Academic)

[32] Li J, Gan R, Guo Q, Liu H, Xu J and Yi F 2018 Tailoring optical responses of infrared plasmonic metamaterial absorbers by optical phonons Opt. Express 26 16769-81

[33] Berk A, Conforti P, Kennett R, Perkins T, Hawes F and Van Den Bosch J 2014 Modtran ${ }^{\circledR}$ 6: a major upgrade of the modtran ${ }^{\circledR}$ radiative transfer code 2014 6th Workshop Hyperspectral Image and Signal Processing: Evolution in Remote Sensing (WHISPERS) (IEEE) pp 1-4

[34] Nielsen M G, Gramotnev D K, Pors A, Albrektsen O and Bozhevolnyi S I 2011 Continuous layer gap plasmon resonators Opt. Express 19 19310-22

[35] Nielsen M G, Pors A, Albrektsen O and Bozhevolnyi S I 2012 Efficient absorption of visible radiation by gap plasmon resonators Opt. Express 20 13311-9

[36] Ding F, Yang Y, Deshpande R A and Bozhevolnyi S I 2018 A review of gap-surface plasmon metasurfaces: fundamentals and applications Nanophotonics 7 1129-56

[37] Deshpande R A, Ding F and Bozhevolnyi S 2019 Dual-band metasurfaces using multiple gap-surface plasmon resonances ACS Appl. Mater. Interfaces 12 1250-6

[38] Jung J, Søndergaard T and Bozhevolnyi S I 2009 Gap plasmon-polariton nanoresonators: scattering enhancement and launching of surface plasmon polaritons Phys. Rev. B 79035401

[39] Omeis F, Smaali R, Gonzalez-Posada F, Cerutti L, Taliercio T and Centeno E 2017 Metal-insulator-metal antennas in the far-infrared range based on highly doped InAsSb Appl. Phys. Lett. 111121108

[40] Lee B J, Wang L P and Zhang Z M 2008 Coherent thermal emission by excitation of magnetic polaritons between periodic strips and a metallic film Opt. Express 16 11328-36

[41] Wang L P and Zhang Z M 2012 Wavelength-selective and diffuse emitter enhanced by magnetic polaritons for thermophotovoltaics Appl. Phys. Lett. 100063902

[42] Ghobadi A, Hajian H, Butun B and Ozbay E 2018 Strong light-matter interaction in lithography-free planar metamaterial perfect absorbers ACS Photonics 5 4203-21

[43] Hanson G W 2008 Dyadic Green's functions and guided surface waves for a surface conductivity model of graphene J. Appl. Phys. 103064302

[44] Gómez-Díaz J S and Perruisseau-Carrier J 2013 Graphene-based plasmonic switches at near infrared frequencies Opt. Express 21 15490-504

[45] Amin M, Farhat M and Bağcı H 2013 A dynamically reconfigurable Fano metamaterial through graphene tuning for switching and sensing applications Sci. Rep. 3 1-8

[46] Wang H and Wang L 2013 Perfect selective metamaterial solar absorbers Opt. Express 21 A1078-A1093

[47] Patel S K, Charola S, Parmar J and Ladumor M 2019 Broadband metasurface solar absorber in the visible and near-infrared region Mater. Res. Express 6086213

[48] Rodrigo D, Limaj O, Janner D, Etezadi D, De Abajo F J G, Pruneri V and Altug H 2015 Mid-infrared plasmonic biosensing with graphene Science 349 165-8

[49] Tong J, Muthee M, Chen S-Y, Yngvesson S K and Yan J 2015 Antenna enhanced graphene $\mathrm{THz}$ emitter and detector Nano Lett. 15 5295-301

[50] Chu H-S and How Gan C 2013 Active plasmonic switching at mid-infrared wavelengths with graphene ribbon arrays Appl. Phys. Lett. 102231107

[51] Mikhailov S A 2013 Graphene-based voltage-tunable coherent terahertz emitter Phys. Rev. B 87115405

[52] Chen P-Y, Argyropoulos C, Farhat M and Gomez-Diaz J S 2017 Flatland plasmonics and nanophotonics based on graphene and beyond Nanophotonics 6 1239-62

[53] Lin I-T, Lai Y-P, Wu K-H and Liu J-M 2014 Terahertz optoelectronic property of graphene: substrate-induced effects on plasmonic characteristics Appl. Sci. 4 28-41

[54] Zhang Y, Li T, Chen Q, Zhang H, O'Hara J F, Abele E and Azad A K 2015 Independently tunable dual-band perfect absorber based on graphene at mid-infrared frequencies $S c i$. Rep. 5 1-8

[55] Lei M, Feng N, Wang Q, Hao Y, Huang S and Bi K 2016 Magnetically tunable metamaterial perfect absorber J. Appl. Phys. 119244504 